\title{
Clinical Observation of Coronary Artery Bypass Grafting with Heart Valve Replacement for Coronary Heart Disease with Heart Valvular Disease
}

ZHOU Xiao-yu, GUO Jiong, SHU Li-liang, XU Jing*

The First Affiliated Hospital of Zhengzhou University

Abstract: Objective: To analyze and explore the clinical observation of coronary artery disease with coronary heart disease and coronary heart disease (CHD) undergoing coronary artery bypass grafting. Methods: From December 2015 to December 2016, 80 patients with coronary heart disease and heart valve were randomly divided into two groups: observation group and control group (40 cases). The observation group was treated with coronary artery bypass grafting combined with simultaneous heart valve replacement. The control group was treated with coronary stent implantation and non-synchronous valve replacement. The improvement of postoperative cardiac function, postoperative hospitalization, adverse events and bridge patency were compared between the two groups. Results: The improvement of cardiac function and the incidence of adverse events were significantly better in the observation group than in the control group $(\mathrm{P}<0.05)$. The time of hospitalization and the time of ventilator were significantly shorter than those of the control group $(\mathrm{P}<0.05)$. The patency rate of the bridge was significantly higher than that of the control group ( $\mathrm{P}$ $<0.05$ ). Conclusion: Coronary artery bypass grafting combined with heart valve replacement in patients with coronary heart disease complicated with valvular heart disease can achieve significant curative effect, which is more ideal and safer than coronary artery bypass surgery combined with non-synchronous valve replacement. The

Key words: coronary heart disease; valvular heart disease; coronary artery bypass grafting; valve replacement; simultaneous surgery

\section{Introduction}

According to the clinical reports show that in recent years the number of aging population in China was significantly

Copyright (C) 2017 ZHOU Xiao-yu, et.al.

doi: http://dx.doi.org/10.18686/aem.v6.88

This is an open-access article distributed under the terms of the Creative Commons Attribution Unported License

(http://creativecommons.org/licenses/by-nc/4.0/), which permits unrestricted use, distribution, and reproduction in any medium, provided the original work is properly cited. 
climbing, followed by the incidence of coronary atherosclerotic heart disease with heart valve disease is also increasing year by year in China, coronary heart disease incidence of mitral or aortic valve disease is about $15 \%$. Such diseases are mainly in elderly and patients with valvular heart disease, the patient is older and sick longer and accompanied by other medical diseases, the condition is relatively complex. At present, patients with coronary heart disease and coronary heart disease underwent coronary artery bypass grafting combined with heart valve surgery, but because of this operation is longer, easy to induce various complications after surgery, thus increasing the operation difficulty and risk, the corresponding clinical reports and research is clearly lacking. However, such surgery can be completed once the treatment, reduce the second operation and reoperation rate, can significantly improve the long-term survival rate of patients. In our hospital, some patients with coronary heart disease with valvular heart disease in the course of treatment of coronary artery bypass grafting in the same period of heart valve replacement surgery, and achieved some research results. The results are summarized as follows.

\section{Materials and methods}

\subsection{General information}

80 patients with coronary heart disease complicated with heart valve were enrolled in our hospital from December 2015 to December 2016. All patients were randomly divided into two groups: 40 patients in each group. There were 21 males and 19 females in the observation group. The age was 45 to 86 years (mean, $64.9 \pm 5.7$ years). The cardiac function grade: grade II, grade III, grade 23, grade 7 . There were 22 males and 18 females in the control group. The age was 46 to 85 years (mean, $65.9 \pm 6.2$ years). The cardiac function grade was 11 cases with grade II, 25 cases of grade III and 4 cases of grade IV. Statistical analysis of the two groups of patients with gender, age, cardiac function classification and other general data difference was not statistically significant $(\mathrm{P}>0.05)$, comparable.

\subsection{Selection criteria}

1) Inclusion criteria: all patients were diagnosed with coronary heart disease with valvular heart disease by ultrasound, coronary angiography and pathology; dynamic electrocardiogram showed left and right bundle branch dysfunction, left ventricular hypertrophy, atrial fibrillation; cardiac function classification in the II IV level; combined with diabetes, hypertension, liver and kidney dysfunction and other diseases; clinical data is complete; better awareness, with the investigation; signed on this study informed consent. 2) Exclusion criteria: suffering from mental illness, lack of autonomy; there are contraindications for surgery; clinical data incomplete; patients and their families did not sign informed consent or can not effectively cooperate with the study.

\subsection{Method}

In the trial group, patients underwent coronary artery bypass grafting for heart valve replacement. The patients were treated with general anesthesia and underwent cardiopulmonary bypass. After successful operation, the incision was performed from the sternum and the heart was exposed. The left internal mammary artery and the left internal mammary artery large saphenous vein free to prepare for surgery. In the aorta and superior vena cava, the inferior vena cava, respectively, intubation to form an extracorporeal circulation. In the course of the application of cold blood cardioplegia intermittent irrigation and coronal venous sinus retrograde perfusion combined with the method of myocardial protection. After the heart to stop the pulse after the first bridge to the distal vascular anastomosis, and then to implement the heart surgery. After the heart is restored to orthosis, the mitral and aortic valve plasty is performed or replaced. The valve is selected for artificial mechanical valve. The mitral valve was replaced by 2-0 Prolene line and the aortic valve was fixed by intermittent stitching. The time of aorta and cardiopulmonary bypass was controlled at (98.18 
$\pm 28.65) \mathrm{min},(163.15 \pm 37.53) \mathrm{min}$. In the control group, patients underwent coronary artery bypass grafting with heart valve replacement. The patients underwent coronary artery bypass grafting, while the time of heart valve operation was controlled at $(216.74 \pm 41.35) \mathrm{d}$. All patients in the group were routinely subjected to hemodynamics and were treated with positive inotropic and vasoactive drugs, reduced pulmonary arterial pressure, expanded coronary artery and underlying disease drug therapy.

\subsection{Observe indicators}

Two groups of patients were followed up for 1 year. (1) Cardiac function improvement index: cardiothoracic ratio, LVEF (left echocardiography fraction), LVD (A-P) (left ventricular end diastolic diameter) (2) Postoperative situation: ICU (severe intensive care ward) monitoring time, the level of heart failure, LVEF (left ventricular ejection fraction), LVD (AP) (left ventricular end diastolic diameter) Ventilator application time, length of stay; (3) Adverse events: flap valve leakage, permanent pacemaker implantation, death and so on. (4) Vascular bridge patency: vascular stenosis diameter $<50 \%$ or no stenosis for the smooth; vascular diameter obstruction $50 \%$ to $99 \%$ of the stenosis; complete occlusion.

\subsection{Statistical methods}

All data were analyzed by SPSS 18.0 statistical software. $(\mathrm{X} \pm \mathrm{s})$ that measurement data, with $\mathrm{t}$ test, count data with $\chi^{2}$ test, compared with the group [n (\%)] said. $\mathrm{P}<0.05$ for the difference was statistically significant.

\section{Results}

\subsection{Heart function improvement}

The improvement of cardiac function in the observation group was significantly better than that in the control group at 1 week and 1 year after operation $(\mathrm{P}<0.05)$. See Table 1 .

Table 1 Comparison of two groups of cardiac function improvement $(\mathrm{x} \pm \mathrm{s})$

\begin{tabular}{|l|l|l|l|l|}
\hline Time & Group & Cholera ratio & LVEF $(\%)$ & LVD $($ A-P $)(\mathrm{mm})$ \\
\hline \multirow{5}{*}{ One week } & $\begin{array}{l}\text { Control group } \\
(\mathrm{n}=40)\end{array}$ & $0.53 \pm 0.08$ & $0.44 \pm 0.13$ & $53.46 \pm 6.42$ \\
\cline { 2 - 6 } & $\begin{array}{l}\text { Observation } \\
\text { group (n=40) }\end{array}$ & $0.70 \pm 0.13$ & $0.57 \pm 0.19$ & $62.81 \pm 7.39$ \\
\cline { 2 - 6 } 1 year & $\mathrm{t}$ & 7.044 & 3.571 & 6.041 \\
\cline { 2 - 6 } & $\mathrm{P}$ & $<0.05$ & $<0.05$ & $<0.05$ \\
\hline \multirow{5}{*}{$\begin{array}{l}\text { Control grouop } \\
(\mathrm{n}=40)\end{array}$} & $0.50 \pm 0.07$ & $0.43 \pm 0.10$ & $51.37 \pm 6.39$ \\
\cline { 2 - 6 } & $\begin{array}{l}\text { Observation } \\
\text { group (n=40) }\end{array}$ & $0.65 \pm 0.12$ & $0.55 \pm 0.21$ & $60.45 \pm 6.95$ \\
\cline { 2 - 5 } & $\mathrm{t}$ & 6.829 & 3.263 & 6.083 \\
\cline { 2 - 5 } & $\mathrm{P}$ & $<0.05$ & $<0.05$ & $<0.05$ \\
\hline
\end{tabular}

\subsection{Postoperative situation}

The time of ICU monitoring, hospitalization and ventilator were significantly shorter in the observation group than in the control group $(\mathrm{P}<0.05)$. See Table 2 . 
Table 2 Comparison of two groups after surgery $(\mathrm{x} \pm \mathrm{s})$

\begin{tabular}{|l|l|l|l|l|}
\hline Group & $\mathrm{n}$ & $\begin{array}{l}\text { ICU monitoring } \\
\text { time } \\
(\mathrm{d})\end{array}$ & $\begin{array}{l}\text { Length of stay } \\
(\mathrm{d})\end{array}$ & $\begin{array}{l}\text { Ventilator } \\
\text { application time } \\
(\mathrm{h})\end{array}$ \\
\hline Control group & 40 & $11.65 \pm 2.53$ & $26.35 \pm 5.24$ & $115.37 \pm 22.42$ \\
\hline $\begin{array}{l}\text { Observational } \\
\text { group }\end{array}$ & 40 & $5.13 \pm 1.24$ & $12.87 \pm 2.46$ & $62.83 \pm 13.41$ \\
\hline $\mathrm{t}$ & & 14.636 & 14.728 & 12.749 \\
\hline $\mathrm{p}$ & & $<0.05$ & $<0.05$ & $<0.05$ \\
\hline
\end{tabular}

\subsection{Comparison of two groups of adverse events}

The incidence of pericardial leakage and the mortality rate were significantly lower than those of the control group ( $\mathrm{P}$ $<0.05)$. See Table 3 .

Table 3

\begin{tabular}{|l|l|l|l|l|}
\hline Group & $\mathrm{N}$ & Flap perimeter & $\begin{array}{l}\text { Permanent } \\
\text { pacemaker } \\
\text { implant }\end{array}$ & Death \\
\hline Control group & 40 & $9(22.50)$ & $11(27.5)$ & $10(25.00)$ \\
\hline $\begin{array}{l}\text { Observational } \\
\text { group }\end{array}$ & 40 & $2(5.00)$ & $6(15.00)$ & $3(7.50)$ \\
\hline $\mathrm{X}^{2}$ & & 5.165 & 1.867 & 4.501 \\
\hline $\mathrm{P}$ & & $<0.05$ & $<0.05$ & $<0.05$ \\
\hline
\end{tabular}

\subsection{Comparison of vascular patency between two groups}

The patency rate of the bridge was $80.00 \%(32 / 40)$ and $95.00 \%(38 / 40)$ in the observation group. The patency rate of the bridge was significantly lower than that of the observation group $(\chi 2=4.114, \mathrm{P}<0.05)$.

\section{Discussion}

Coronary heart disease and valvular heart disease are common clinical cardiovascular disease, coronary heart disease is caused by coronary atherosclerosis stenosis, obstruction, myocardial ischemia caused by hypoxic necrosis of heart disease; heart valve disease refers to the aorta flap, mitral valve and other valves due to degenerative changes, rheumatic fever, mucous degeneration, ischemic necrosis and other effects of blood flow, leading to cardiac dysfunction, and ultimately cause valvular disease. Coronary heart disease with valvular heart disease is common in myocardial infarction after the occurrence of valvular ischemic degeneration in patients with a combination of the two can significantly improve the risk of disease, the safety of patients poses a serious threat.

Coronary atherosclerotic heart disease (coronary heart disease) with heart valve disease patients, surgical treatment required at the same time coronary artery bypass grafting $(\mathrm{CABG})$ and heart valve surgery, due to poor preoperative cardiac function, surgical operation complex and long operative time, making the risk of surgery significantly higher than the line of simple CABG or simple heart valve surgery. According to foreign literature, the early mortality rate of such surgery was $7 \%-24 \%$. 
Domestic literature reported that $12.68 \%$ of rheumatic heart disease patients with coronary heart disease. Patients before surgery, comprehensive and detailed diagnosis and treatment is particularly important, not only determines the surgical operation, but also determines the long-term prognosis after surgery. In general, patients with rheumatic valvular disease, if the age of $>50$ years old, previous angina or related medical history, ECG examination of myocardial ischemia (such as changes in ST segment) or other coronary heart disease risk factors (such as high Blood pressure, diabetes, hyperlipidemia, smoking, etc.), to meet one of the above factors in patients with preoperative routine CAG should be checked to understand whether the merger of coronary artery lesions and lesions of the specific circumstances, according to the development of better surgical program.

Complete revascularization is an important guarantee for successful surgery. DiSesa et al. reported an early death rate of only $6.3 \%$ in 85 patients with complete revascularization of CABG + MVR myocardium, compared with $23.8 \%$ in 21 patients with incomplete $\mathrm{CABG}+\mathrm{MVR}$ myocardial revascularization. Different patients, appropriate surgical techniques and artificial blood vessels, to ensure smooth vascular bridge, complete revascularization of myocardium, as much as possible to restore the blood supply to the heart, improve the impact of hemodynamics. To correct the correct operation of the anastomosis of the coronary artery, the anastomosis position is accurate, and the time of occlusion and extracorporeal circulation is shortened as far as possible under the premise of convenience, fastness and simplicity.

Coronary heart disease with valvular heart disease patients in the course of treatment of coronary artery bypass grafting simultaneous treatment of heart valve replacement, due to poor preoperative cardiac function, surgical operation process is more complex, intraoperative long time and other factors. Its surgical risk and simple coronary artery bypass grafting and simple heart valve replacement compared to significantly increased. Coronary heart disease caused by myocardial ischemia can cause valvular tendon ring and papillary muscle dysfunction, induced valvular disease. Coronary heart disease at the same time will reduce the patient's myocardial contractility, change the internal structure and function of the ventricle, and further aggravate the patient's condition. Current clinical studies have shown that patients with coronary artery disease associated with valvular heart disease may be treated with coronary artery bypass grafting for simultaneous cardiac valve replacement. When patients with coronary artery disease and heart valve disease coexist at the same time, regardless of whether the presence of myocardial insufficiency in patients with performance, this time should be treated on coronary artery disease, and nearly half of patients in the heart valve surgery at the same time need to be coronary artery Road transplantation. This will not only reduce the risk of secondary surgery, but also reduce the repeated surgery to patients with trauma and pain, reduce treatment time, increase the patient's perioperative cardiac function recovery. But the clinical lack of a large number of coronary artery disease with heart valve disease with the clinical basis of surgery, so the design of the study. The success of the surgery depends on whether the intraoperative myocardial protection is in place.

The protective measures taken during the course of the study will help to reduce pulmonary edema and protect the postoperative cardiac function, and should be based on the patient's pathophysiology according to the implementation of targeted treatment measures, which reduce the mortality rate and the incidence of complications is of great significance, $(\mathrm{P}<0.05)$. In the experimental group, the patients' postoperative cardiac function indexes and the control group were significantly higher than those in the control group $(\mathrm{P}<0.05)$, and the difference between the two groups was statistically significant $(\mathrm{P}<0.05)(\mathrm{P}<0.05)$. The difference between the two groups was statistically significant $(\mathrm{P}$ $<0.05$ ). This shows that the clinical efficacy of surgery over the same period more significant, especially to improve cardiac function, reduce postoperative mortality and so on.

In summary, coronary artery bypass grafting combined with heart valve replacement in the treatment of coronary heart disease with valvular heart disease is feasible and safe, can effectively improve the patient's heart function, and shorten the hospital stay. But the perioperative period must take appropriate symptomatic treatment measures to maximize the 
protection of surgical safety.

\section{References}

1. Wang Wen Rui, Gao Chang Qing, Li Bo Jun, Wu Yang, Wang Rong, Xiao Cun Song, Sheng Wei. Heart Valve Replacement with Coronary Artery Bypass Grafting in 107 Cases of Clinical Analysis [J]. Medical Journal, 2012,07: 51-54.

2. Li Mei Sen, Zheng Bao Shi. Heart Valve Replacement Surgery in the Same Period of Coronary Artery Bypass Graft Surgery Nursing [J]. Minimally Invasive Medicine, 2014,05: 624-625 +621.

3. Zhang Guo Xin, Qiao Gang, Xia Dong Sheng. Coronary Artery Bypass Grafting in the Treatment of Coronary Heart Disease with Heart Valve Disease by Heart Valve Surgery [J]. Chinese Journal of Clinical Medicine, 2015,26: 74-75 +80 The

4. Wang Wei Ling. Coronary Artery Bypass Grafting Valve Replacement Surgery [J]. General Nursing, 2011,32: 2955-2956.

5. Wang Yan. Coronary Artery Bypass Grafting In The Same Period Of Heart Valve Replacement Surgery [J]. Zhe Jiang Medical, 2015,09: 801-803.

6. Yu Bo, Liu Jin Ping, Qin Liang Guang, Chen Hong Ye, Tan Qi Ming. Heart Valve Surgery in The Same Period of Coronary Artery Bypass Grafting in 19 Cases [J]. Huai Hai Medicine, 2009,02: 131-132. 